\title{
Tilted subwavelength gratings: controlling anisotropy in metamaterial nanophotonic waveguides
}

\author{
José Manuel luque-González ${ }^{1 *}$, Alaine Herrero-Bermello ${ }^{2}$, Alejandro \\ Ortega-MoñuX ${ }^{1}$, Íñigo Molina-Fernández ${ }^{1,3}$, Aitor V. Velasco ${ }^{2}$, Pavel \\ Cheben $^{4}$, Jens H. Schimd ${ }^{4}$, Shurui WAng ${ }^{4}$, And Robert HaliR ${ }^{1,3}$ \\ ${ }^{1}$ Universidad de Málaga, Dpto. de Ingeniería de Comunicaciones, ETSI Telecomunicación, Campus de Teatinos s/n, 29071 Málaga, Spain. \\ ${ }^{2}$ Institute of Optics, Spanish National Research Council, 28006 Madrid, Spain \\ ${ }^{3}$ Bionand Center for Nanomedicine and Biotechnology, Parque Tecnológico de Andalucía, 29590 Málaga, España \\ ${ }^{4}$ National Research Council Canada, 1200 Montreal Road, Bldg. M50, Ottawa K1A 0R6, Canada. \\ *Corresponding author: jmlg@ic.uma.com
}

Compiled June 18, 2018

\begin{abstract}
Subwavelength grating (SWG) structures are an essential tool in silicon photonics, enabling the synthesis of practical metamaterials with controllable refractive index. Here we propose, for the first time, tilting the grating elements to gain control over the anisotropy of the metamaterial. Rigorous FDTD simulations demonstrate that a $45^{\circ}$ tilt results in an effective index variation on the fundamental TE mode of 0.23 refractive index units, whereas the change in the TM mode is 20 times smaller. Our simulation predictions are corroborated by experimental results. We furthemore propose an accurate theoretical model for designing tilted SWG structures based on rotated uniaxial crystals, which is functional over a wide wavelength range and for both the fundamental and higher order modes. The proposed structures open up promising venues in polarization management of silicon photonic devices. (C) 2018 Optical Society of America under the terms of the OSA Open Access Publishing Agreement
\end{abstract}

http://dx.doi.org/10.1364/optica.XX.XXXXXX

\section{INTRODUCTION}

Periodic laminar structures with a period $(\Lambda)$ much smaller than the wavelength of propagating light $(\lambda)$ behave, with respect to the average electromagnetic field, as homogeneous metamaterials [1]. This homogenized metamaterial is frequently characterized as an isotropic medium with a different equivalent refractive index for each polarization, or more generally, as a form birefringent anisotropic medium [2]. These approaches have long been studied in free-space optics [3, 4], enabling, for example, the design of half-wave and quarter-wave plates $[5,6]$.

More recently, metamaterial nanostructures have been incorporated into silicon-on-insulator (SOI) waveguides, in the form of subwavelength gratings (SWG) [7-9]. In SWG devices, a controllable refractive index is synthesized by adjusting the relative amount of silicon per period, i.e. the duty cycle $(D C)$. By approximating the SWG as an isotropic homogeneous material, a wide range of high-performance integrated devices has been demonstrated, including fiber-to-chip grating couplers [10-13], edge couplers $[7,14,15]$, waveguide couplers [16, 17], bragg filters [18, 19], and evanescent field waveguide sensors [20, 21]. Simple anisotropic models have enabled further applications of SWG waveguides, such as ultra-broadband devices [22, 23], polarization management [24, 25], or evanescent field confinement $[26,27]$. However, duty cycle changes affect both polarizations [1], and can also hamper device fabrication due to aspect ratio dependent etching and proximity effects [28].

In this work, we propose, analyze and experimentally demonstrate a technique to control the anisotropy of an SWG structure. We show that tilting the silicon segments, as shown in Fig. 1, mainly affects the in-plane (TE) modes, with little impact on the out-of-plane (TM) modes, while maintaining a constant duty 


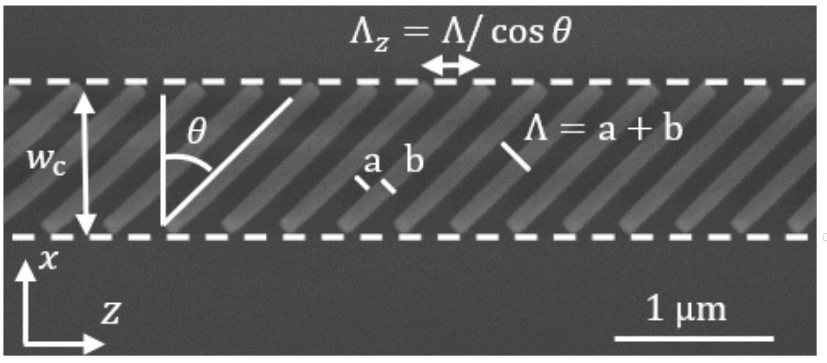

Fig. 1. Scanning Electron Microscope (SEM) image of a fabricated tilted SWG waveguide prior to the deposition of the $\mathrm{SiO}_{2}$ cladding. $w_{c}=1 \mu \mathrm{m}, \Lambda=0.25 \mu \mathrm{m}, D C=0.5, \theta=45^{\circ}$.

cycle and minimum feature size.

\section{REVISITING LAMINAR PERIODIC STRUCTURES}

In order to gain insight into the physics of tilted SWG structures, let us first revisit the properties of a laminar periodic structure. Specifically, consider a periodic structure with period $\Lambda$, composed of two transversally-infinite materials with refractive indexes $n_{1}$ and $n_{2}$, and thicknesses $a=D C \cdot \Lambda$ and $b=(1-D C) \cdot \Lambda$, respectively, as shown in Fig. 2(a). For the purpose of illustration, typical values for SWG waveguides in the SOI platform are considered, i.e. $\Lambda=0.2 \mu \mathrm{m}, D C=0.5$, $n_{1}=3.476, n_{2}=1.444$ and $\lambda_{0}=1.55 \mu \mathrm{m}$. A plane wave propagating along this structure with an angle $\phi$ respect to the $z$ axis is described by its $k$-vector $\vec{k}(\phi)=k_{x} \hat{x}+k_{z} \hat{z}$, where $\phi=\operatorname{atan}\left(k_{x} / k_{z}\right)$ and $k_{x}, k_{z}$ are related via the following dispersion equation [29]:

$$
\cos \left(k_{z} \Lambda\right)=\cos \left(k_{1 x} a\right) \cos \left(k_{2 x} b\right)-\Delta \sin \left(k_{1 x} a\right) \sin \left(k_{2 x} b\right),
$$

where $k_{i x}=\sqrt{\left(k_{0} n_{i}\right)^{2}-k_{x}^{2}}, k_{0}=2 \pi / \lambda_{0}$ and $\Delta$ is a polarizationdependent factor. Defining as TE polarization the plane waves with electric field polarized in the $x$ - $z$ plane, and as TM polarization the y-polarized waves, we have:

$$
\Delta_{T E}=\frac{1}{2}\left(\frac{n_{2}^{2}}{n_{1}^{2}} \frac{k_{1 x}}{k_{2 x}}+\frac{n_{1}^{2}}{n_{2}^{2}} \frac{k_{2 x}}{k_{1 x}}\right) \text { and } \Delta_{T M}=\frac{1}{2}\left(\frac{k_{1 x}}{k_{2 x}}+\frac{k_{2 x}}{k_{1 x}}\right) .
$$

The normalized magnitude of the $k$-vector, $|\vec{k}(\phi)| / k_{0}$ calculated through Eq. (1), is shown with solid lines in Fig. 2(c) for both polarizations. For a TM ( $y$-polarized) plane wave, the magnitude of the $k$-vector is practically independent of the angle $\phi$ (red solid curve), because its electric field is orientated parallel to the periodic interfaces. However, for a TE plane wave, it strongly depends on the angle $\phi$ (blue solid curve), because the electric field orientation changes from parallel to the interfaces when propagating along $z$ axis, to normal to the interfaces when the propagation is along $x$ direction. Therefore, the normalized magnitude of the $k$-vector in a TE plane wave changes from $n_{x x}=|\vec{k}(0)| / k_{0}$ to $n_{z z}=|\vec{k}(90)| / k_{0}$ when the propagation direction changes from $z$ to $x$.

This behavior is closely related to that of a homogeneous but anisotropic uniaxial medium described by the refractive index tensor $\mathbf{n}=\operatorname{diag}\left[n_{x x}, n_{y y}, n_{z z}\right]$, illustrated in Fig. 2(b). For the aforementioned parameters typical for silicon SWG waveguides, we obtain $n_{x x}=2.79$ and $n_{z z}=1.94$, where $n_{x x}=n_{y y}$ from symmetry considerations. The $k$-vector of a plane wave propagating

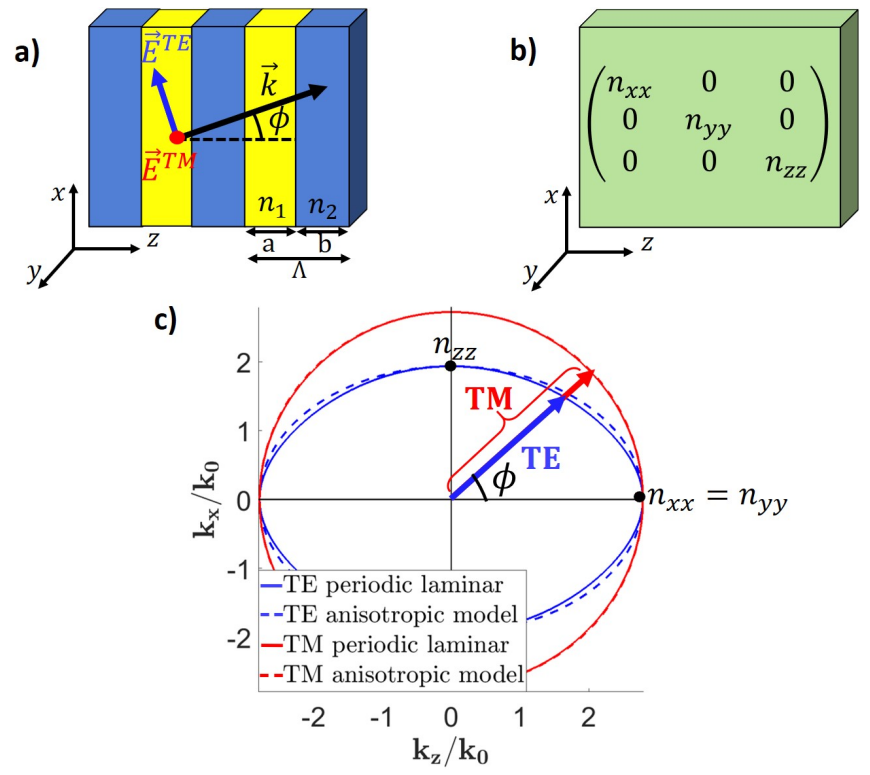

Fig. 2. a) Laminar periodic structure comprising two materials with refractive indexes $n_{1}$ and $n_{2}$ and thicknesses $a=D C \cdot \Lambda$ and $b=(1-D C) \cdot \Lambda . b)$ Anisotropic material described by the tensor $\mathbf{n}=\operatorname{diag}\left[n_{x x}, n_{y y}, n_{z z}\right]$, which models the propagation properties of a laminar periodic structure. c) Comparison, for both polarizations, of the $k$-vector of a laminar structure and an anisotropic homogeneous metamaterial for: $n_{1}=3.476$, $n_{2}=1.444, \Lambda=0.2 \mu \mathrm{m}, D C=0.5$ and $\lambda_{0}=1.55 \mu \mathrm{m}$.

through such a homogeneous anisotropic uniaxial medium can be calculated using the well-known dispersion equations:

$$
\left(\frac{k_{z}}{n_{x x}}\right)^{2}+\left(\frac{k_{x}}{n_{z z}}\right)^{2}=k_{0}^{2} \text { and }\left(\frac{k_{z}}{n_{y y}}\right)^{2}+\left(\frac{k_{x}}{n_{y y}}\right)^{2}=k_{0}^{2}
$$

for TE and TM polarization respectively [29]. From Fig. 2(c) it is clear that this anisotropic homogenization, shown with dashed lines, provides an excellent approximation of the laminar periodic structure. In other words, the laminar periodic structure [Fig. 2(a)] works as a uniaxial crystal [Fig. 2(b)].

\section{TILTED SWG WAVEGUIDES}

We will now show that this behavior can be extended to SWG waveguides, where the periodic structure is finite in the transversal directions [Fig. 3(a)]. Note that when tilting the segments in the SWG waveguide, the period along the propagation direction ( $\left.\Lambda_{z}\right)$ is given by $\Lambda_{z}=\Lambda / \cos (\theta)$. Extending the equivalence between a periodic structure and a uniaxial crystal, we model the tilted SWG waveguide [Fig. 3(a)] as an anisotropic homogeneous waveguide [Fig. 3(b)]. With the coordinate system set by the light propagation along the waveguide ( $z$-direction), the tilt angle $\theta$ of the SWG segments results in a rotation of the diagonal index tensor, yielding the non-diagonal index tensor $\tilde{\mathbf{n}}$ [30]:

$$
\tilde{\mathbf{n}}(\theta)=\mathbf{T}^{-\mathbf{1}}(\theta) \mathbf{n} \mathbf{T}(\theta)=\left[\begin{array}{ccc}
\tilde{n}_{x x}(\theta) & 0 & \tilde{n}_{x z}(\theta) \\
0 & \tilde{n}_{y y} & 0 \\
\tilde{n}_{x z}(\theta) & 0 & \tilde{n}_{z z}(\theta)
\end{array}\right],
$$


where $\mathbf{T}$ is the rotation matrix in the $x-z$ plane and the different elements of the tensor $\tilde{\mathbf{n}}$ are give by:

$$
\begin{aligned}
& \tilde{n}_{x x}^{2}=n_{x x}^{2} \cos ^{2}(\theta)+n_{z z}^{2} \sin ^{2}(\theta), \\
& \tilde{n}_{y y}=n_{y y}, \\
& \tilde{n}_{z z}^{2}=n_{x x}^{2} \sin ^{2}(\theta)+n_{z z}^{2} \cos ^{2}(\theta), \\
& \tilde{n}_{x z}^{2}=\left(n_{z z}^{2}-n_{x x}^{2}\right) \cos (\theta) \sin (\theta) .
\end{aligned}
$$

In order to validate this model, we compare the modes guided by the structures shown in Fig. 3(a) and Fig. 3(b) for a multimode waveguide $\left(w_{c}=3.25 \mu \mathrm{m}, \Lambda=0.2 \mu \mathrm{m}, D C=0.5\right)$ in a standard $220 \mathrm{~nm}$ thick silicon waveguide. First, we compare, in Fig. 3(c) and Fig. 3(d), the electric field of the fundamental TE mode propagating through the periodic and homogeneous waveguide for a tilt angle $\theta=30^{\circ}$. As expected, the average electric field is very similar in both cases. Note that tilting the SWG segments implies a phase front tilt which is predicted by the uniaxial crystal model [30].

We then calculate the effective indexes, $\left(n_{\text {eff }}\right)$, of the fundamental TE and TM modes of the anisotropic homogeneous waveguide for tilt angles ranging from $\theta=0^{\circ}$ to $\theta=45^{\circ}$. In Fig. $3(\mathrm{e})$ we compare these results with rigorous FDTD simulations of
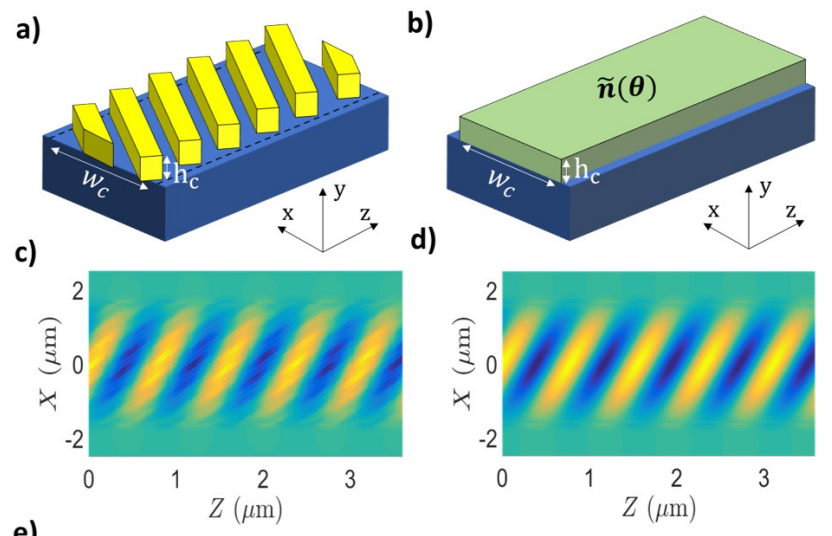

e)

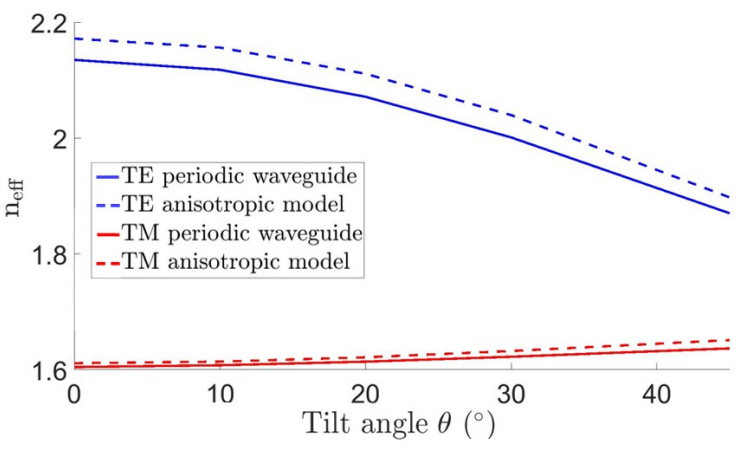

Fig. 3. a) Schematic of a tilted SWG multimode waveguide and $b$ ) the corresponding homogeneous anisotropic model. For the sake of clarity the $\mathrm{SiO}_{2}$ upper cladding is not drawn. c) Real part of the main component $\left(E_{x}\right)$ of the fundamental TE mode propagating along a SWG waveguide with a $\theta=30^{\circ}$ tilt angle at $\lambda_{0}=1.55 \mu \mathrm{m}$. d) Real part of the main component $\left(E_{x}\right)$ of the fundamental TE mode propagating along the homogeneous anisotropic waveguide at $\lambda_{0}=1.55 \mu \mathrm{m}$. e) Effective index of the fundamental TE and TM modes of the tilted SWG waveguide and the anisotropic homogeneous waveguide at $\lambda_{0}=1.55 \mu \mathrm{m}$. a)

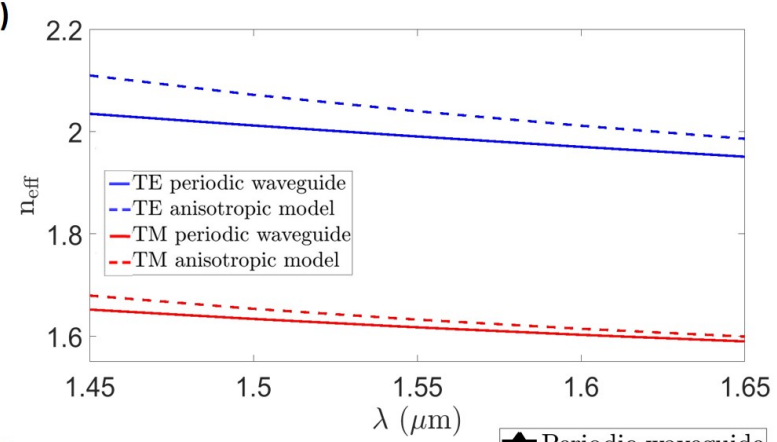

b)

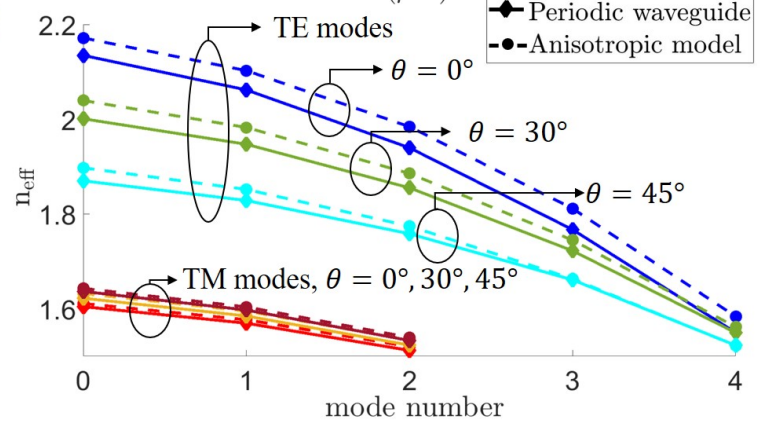

Fig. 4. a) Effective index of the fundamental TE and TM mode of the $\theta=30^{\circ}$ tilted multimode SWG waveguide as a function of wavelength. b) Effective indices of guided modes in the multimode SWG waveguide, for both polarization, and different tilt angles $\left(\lambda_{0}=1.55 \mu \mathrm{m}\right)$.

the effective indices of the Floquet modes of the tilted SWG waveguide calculated following the method outlined in [20]. From this comparison we obtain two of the main results reported in this paper: First, the effective index of the TE mode changes significantly with the tilt angle, achieving a refractive index variation of $\Delta \mathrm{n}_{\text {eff }}^{\mathrm{TE}} \sim 0.25$ for $\theta=45^{\circ}$ whereas the effective index of the TM mode remains practically constant $\left(\Delta \mathrm{n}_{\text {eff }}^{\mathrm{TM}} \sim 0.02\right)$ for the same structure. This enables engineering of the metamaterial anisotropy and the effective refractive index while maintaining a constant duty cycle and minimum feature size. Second, the anisotropic homogeneous model exhibits the same behavior as the periodic structure when tilting the SWG segments, providing a quick yet accurate tool to perform preliminary designs.

From Eq. (1) we observe that the laminar periodic structure is dispersive even if the constituent materials are not. If this structural dispersion is taken into account, the model also predicts the wavelength dependence of the periodic structure, as shown in Fig. 4(a) for an example of $\theta=30^{\circ}$. Examining this wavelength behavior reveals the intrinsic limitation of any homogenizationbased model: as the laminar periodic structure works closer to the Bragg resonance $\left(\lambda_{\text {Bragg }} \sim 1.3 \mu \mathrm{m}\right)$, accuracy is progressively reduced. Finally, Fig. 4(b) shows the effective indexes of the guided TE and TM modes for different tilt angles, revealing that the anisotropic homogenization also provides a good estimation for the higher order modes.

\section{EXPERIMENTAL RESULTS}

To experimentally validate the proposed structures a set of tilted SWG waveguides was fabricated in a $220 \mathrm{~nm}$ SOI platform with $3 \mu \mathrm{m}$ buried oxide (BOX). A $3 \mu \mathrm{m}$ thick upper $\mathrm{SiO}_{2}$ cladding was deposited by plasma enhanced chemical vapor deposition 
(PECVD). The test structure was a Mach-Zehnder Interferometer (MZI) where a tilted SWG waveguide was placed in one arm and a reference straight SWG waveguide was placed in the other arm [Fig. 5(a)]. This architecture allows us to easily measure the group index difference between these two types of SWG waveguides. The tapers between the homogeneous and SWG waveguides in the MZI arms were $30 \mu \mathrm{m}$ long to avoid transition losses, and the SWG waveguides were $600 \mu \mathrm{m}$ long to accurately characterize the group index variation; a period of $\Lambda=0.25 \mu \mathrm{m}$ was used. To reduce the influence of fabrication jitter, narrow single-mode SWG waveguides are preferred [31], so a width of $w_{c}=1 \mu \mathrm{m}$ was chosen. Highly-efficient input and output SWG edge couplers $[7,14]$ were used to couple light in and out of the chip with a lensed polarization maintaining fiber (PMF). The transmission spectrum of each MZI [Fig. 5(b), inset] was measured by sweeping the wavelength of a tunable laser while measuring output power with a power meter. From the measured transmittance function the group index difference $\left(\Delta \mathrm{n}_{\mathrm{g}}\right)$ is calculated using the procedure described in [32]. A comparison with the FDTD simulation results [Fig. 5(b)] shows an excellent agreement for both polarization. By simulating the relation between the group index and effective index in both waveguides, the effective index difference can be estimated from the measured group index difference. In particular, for the fabricated single-mode $45^{\circ}$ tilt SWG waveguide we find an effective index variation of $\Delta \mathrm{n}_{\text {eff }}^{\mathrm{TE}} \sim 0.23$ for the TE mode and only $\Delta \mathrm{n}_{\mathrm{eff}}^{\mathrm{TM}} \sim 0.01$ for the TM mode.

\section{CONCLUSIONS}

In conclusion, we have shown through both simulation and experiments that tilted SWG structures enable engineering of the effective metamaterial anisotropy in nanophotonics waveguides. Tilting the SWG segments strongly affects both fundamental and higher order TE modes, while this effect is significantly less prominent for TM modes. This approach provides a new degree of freedom to engineer refractive index in silicon waveguides, while circumventing small variable duty cycles that hamper fabrication. Furthermore, a simple yet accurate homogenization model, based on Eqs. (1)-(5), has been developed and used to characterize and design these structures. The control over metamaterial anisotropy provided by this approach offers promising prospects for advanced polarization management in nanophotonics waveguides.

\section{ACKNOWLEDGMENTS}

We would like to thank Jean Lapointe for the e-beam processing.

\section{FUNDING}

We acknowledge funding from: Universidad de Málaga; Ministerio de Economía y Competitividad (MINECO) (TEC201680718-R, TEC2015-71127-C2-R, IJCI-2016-30484); Ministerio de Educación, Cultura y Deporte (MECD) (FPU16/06762); Fondo Europeo de Desarrollo Regional-FEDER; Comunidad de Madrid (SINFOTON-CM S2013/MIT-2790); EURAMET (JRP-i22 14IND13 Photind, H2020-MSCA-RISE-2015:SENSIBLE).

\section{REFERENCES}

1. S. M. Rytov, "Electromagnetic properties of a finely stratified medium," Sov. Phys. JETP 2, 466 (1956).
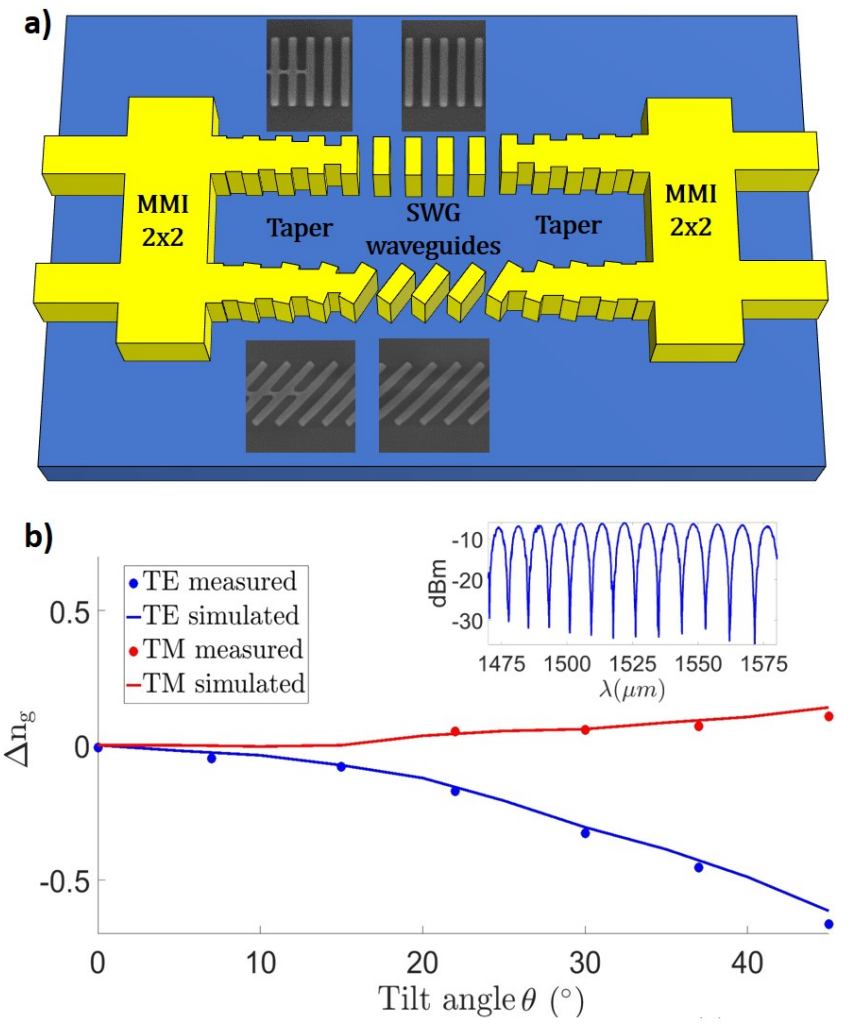

Fig. 5. a) Schematic of the Mach-Zehnder interferometer used for the characterization of the tilted SWG waveguides. Insets: SEM images of the SWG tapers and waveguides. b) Measured and simulated group index difference between the waveguides in the MZI arms. Inset: MZI transmittance measurement for a $\theta=37^{\circ}$ tilt angle.

2. M. Born and E. Wolf, Principles of optics (Cambridge University Press, 2013), chap. 14.

3. H. Kikuta, H. Yoshida, and K. Iwata, "Ability and limitation of effective medium theory for subwavelength gratings," Opt. Rev. 2, 92 (1995).

4. P. Lalanne and D. Lemercier-Lalanne, "On the effective medium theory of subwavelength periodic structures," J. Mod. Opt. 43, 2063 (1996).

5. D. C. Flanders, "Submicrometer periodicity gratings as artificial anisotropic dielectrics," Appl. Phys. Lett. 42, 492 (1983).

6. H. Kikuta, Y. Ohira, and K. Iwata, "Achromatic quarterwave plates using the dispersion of form birefringence," Appl. Opt. 36, 1566 (1997).

7. P. Cheben, D.-X. Xu, S. Janz, and A. Densmore, "Subwavelength waveguide grating for mode conversion and light coupling in integrated optics," Opt. Express 14, 4695 (2006).

8. R. Halir, P. J. Bock, P. Cheben, A. Ortega-Moñux, C. AlonsoRamos, J. H. Schmid, J. Lapointe, D.-X. Xu, J. G. Wangüemert-Pérez, Í. Molina-Fernández, and S. Janz, “Waveguide sub-wavelength structures: a review of principles and applications," Laser Photonics Rev. 9, 25-49 (2014).

9. R. Halir, A. Ortega-Moñux, D. Benedikovic, G. Z. Mashanovich, J. G. Wangüemert-Pérez, J. H. Schmid, Íñigo Molina-Fernández, and P. Cheben, "Subwavelengthgrating metamaterial structures for silicon photonic devices," Proc. IEEE (2018). To be published. 
10. X. Chen and H. K. Tsang, "Polarization-independent grating couplers for silicon-on-insulator nanophotonic waveguides," Opt. Lett. 36, 796 (2011).

11. X. Chen, K. Xu, Z. Cheng, C. K. Y. Fung, and H. K. Tsang, "Wideband subwavelength gratings for coupling between silicon-on-insulator waveguides and optical fibers," Opt. Lett. 37, 3483 (2012).

12. Y. Wang, W. Shi, X. Wang, Z. Lu, M. Caverley, R. Bojko, L. Chrostowski, and N. A. F. Jaeger, "Design of broadband subwavelength grating couplers with low back reflection," Opt. Lett. 40, 4647 (2015).

13. A. Sánchez-Postigo, J. G. Wangüemert-Pérez, J. M. LuqueGonzález, Í. Molina-Fernández, P. Cheben, C. A. AlonsoRamos, R. Halir, J. H. Schmid, and A. Ortega-Moñux, "Broadband fiber-chip zero-order surface grating coupler with $0.4 \mathrm{db}$ efficiency," Opt. Lett. 41, 3013 (2016).

14. P. Cheben, J. H. Schmid, S. Wang, D.-X. Xu, M. Vachon, S. Janz, J. Lapointe, Y. Painchaud, and M.-J. Picard, "Broadband polarization independent nanophotonic coupler for silicon waveguides with ultra-high efficiency," Opt. Express 23, 22553 (2015).

15. T. Barwicz, Y. Taira, T. W. Lichoulas, N. Boyer, Y. Martin, H. Numata, J. W. Nah, S. Takenobu, A. Janta-Polczynski, E. L. Kimbrell, R. Leidy, M. H. Khater, S. Kamlapurkar, S. Engelmann, Y. A. Vlasov, and P. Fortier, "A novel approach to photonic packaging leveraging existing highthroughput microelectronic facilities," IEEE J. Sel. Top. Quantum Electron. 22, 455-466 (2016).

16. A. Ortega-Moñux, C. Alonso-Ramos, A. Maese-Novo, R. Halir, L. Zavargo-Peche, D. Pérez-Galacho, Íñigo MolinaFernández, J. G. Wangüemert-Pérez, P. Cheben, J. H. Schmid, J. Lapointe, D. Xu, and S. Janz, "An ultra-compact multimode interference coupler with a subwavelength grating slot," Laser \& Photonics Rev. 7, 12 (2013).

17. B. Naghdi and L. R. Chen, "Spectral engineering of subwavelength-grating-based contradirectional couplers," Opt. Express 25, 25310 (2017).

18. D. Pérez-Galacho, C. Alonso-Ramos, F. Mazeas, X. L. Roux, D. Oser, W. Zhang, D. Marris-Morini, L. Labonté, S. Tanzilli, É. Cassan, and L. Vivien, “Optical pump-rejection filter based on silicon sub-wavelength engineered photonic structures," Opt. Lett. 42, 1468 (2017).

19. J. Čtyroký, J. G. Wangüemert-Pérez, P. Kwiecien, I. Richter, J. Litvik, J. H. Schmid, Í. Molina-Fernández, A. OrtegaMoñux, M. Dado, and P. Cheben, "Design of narrowband bragg spectral filters in subwavelength grating metamaterial waveguides," Opt. Express 26, 179 (2018).

20. J. G. Wangüemert-Pérez, P. Cheben, A. Ortega-Moñux, C. Alonso-Ramos, D. Pérez-Galacho, R. Halir, I. MolinaFernández, D.-X. Xu, and J. H. Schmid, “Evanescent field waveguide sensing with subwavelength grating structures in silicon-on-insulator," Opt. Lett. 39, 4442 (2014).

21. J. Flueckiger, S. Schmidt, V. Donzella, A. Sherwali, D. M. Ratner, L. Chrostowski, and K. C. Cheung, "Subwavelength grating for enhanced ring resonator biosensor," Opt. express 24, 15672 (2016).

22. R. Halir, P. Cheben, J. M. Luque-González, J. D. SarmientoMerenguel, J. H. Schmid, G. Wangüemert-Pérez, D.-X. Xu, S. Wang, A. Ortega-Moñux, and Í. Molina-Fernández, "Ultra-broadband nanophotonic beamsplitter using an anisotropic sub-wavelength metamaterial," Laser Photonics Rev. 10, 1039 (2016).

23. D. González-Andrade, J. G. Wangüemert-Pérez, A. V. Ve- lasco, A. Ortega-Moñux, A. Herrero-Bermello, I. MolinaFernández, R. Halir, and P. Cheben, "Ultra-broadband mode converter and multiplexer based on sub-wavelength structures," IEEE Photonics J. 10, 1 (2018).

24. H. Nikkhah, M. Hasan, and T. Hall, "The talbot effect in a metamaterial," Appl. Phys. A 124, 106 (2018).

25. L. Xu, Y. Wang, A. Kumar, D. Patel, E. El-Fiky, Z. Xing, R. Li, and D. V. Plant, "Polarization beam splitter based on mmi coupler with swg birefringence engineering on soi," IEEE Photonics Technol. Lett. 30, 403 (2018).

26. S. Jahani and Z. Jacob, "Transparent subdiffraction optics: nanoscale light confinement without metal," Optica. 1, 96 (2014).

27. S. Jahani, S. Kim, J. Atkinson, J. C. Wirth, F. Kalhor, A. A. Noman, W. D. Newman, P. Shekhar, K. Han, V. Van, R. G. DeCorby, L. Chrostowski, M. Qi, and Z. Jacob, "Controlling evanescent waves using silicon photonic all-dielectric metamaterials for dense integration," Nat. Commun. 9, 1893 (2018).

28. Y. Tang, Z. Wang, L. Wosinski, U. Westergren, and S. He, "Highly efficient nonuniform grating coupler for silicon-oninsulator nanophotonic circuits," Opt. Lett. 35, 1290 (2010).

29. P. Yeh, A. Yariv, and C.-S. Hong, "Electromagnetic propagation in periodic stratified media. i. general theory," J. Opt. Soc. Am. 67, 423 (1977).

30. D. Marcuse, "Modes of a symmetric slab optical waveguide in birefringent media-part i: Optical axis not in plane of slab," IEEE J. Quantum Electron. 14, 736 (1978).

31. A. Ortega-Moñux, J. Čtyrokỳ, P. Cheben, J. H. Schmid, S. Wang, Í. Molina-Fernández, and R. Halir, "Disorder effects in subwavelength grating metamaterial waveguides," Opt. Express 25, 12222 (2017).

32. J. D. Sarmiento-Merenguel, A. Ortega-Moñux, J.-M. Fédéli, J. G. Wangüemert-Pérez, C. Alonso-Ramos, E. DuránValdeiglesias, P. Cheben, Í. Molina-Fernández, and R. Halir, "Controlling leakage losses in subwavelength grating silicon metamaterial waveguides," Opt. Lett. 41, 3443 (2016). 